\title{
Metodología, técnicas e instrumentos para la explotación demográfica de los registros parroquiales
}

Methodology, techniques and instruments for the demographical exploitation of parish records

\section{Ana María PRIETo GaRcía}

Facultad de Filosofía y Letras, Universidad de Extremadura, España, any_751@hotmail.com

\begin{abstract}
Resumen
Se pretende mostrar la utilidad e importancia de los registros parroquiales para los trabajos de carácter histórico-demográficos, así como ofrecer una visión general de los criterios metodológicos y los instrumentos necesarios para extraer su información con la finalidad de elaborar un estudio sobre la evolución de la población. Para ello, se presentan los posibles métodos de explotación de las fuentes, las técnicas de análisis de contenido y los programas informáticos fundamentales para su desarrollo.
\end{abstract}

Palabras clave: Demografía. Libros parroquiales. Análisis de registros. Bases de datos. Cáceres (España). Extremadura (España).

\section{Introducción}

La demografía histórica emergió como una disciplina autónoma en la década de 1950, centrada, fundamentalmente, en el estudio del comportamiento demográfico de las poblaciones pre-modernas (1). Desde su surgimiento, siempre se ha intentado innovar en los métodos, técnicas e instrumentos que permitan obtener unas medidas sofisticadas y un análisis más exhaustivo. Asimismo, este interés de renovación radica, en parte, en la necesidad de los historiadores de obtener unas medidas cuantitativas de los acontecimientos pasados que les permita esclarecer racionalmente un hecho $y$ hallar una explicación.

En este sentido, el propósito de este trabajo es señalar, a grandes rasgos, la necesidad de contar con unas buenas fuentes y una metodología adecuada para conseguir elaborar un trabajo histórico-demográfico. La carencia de este tipo de estudios en los que se describan detalladamente los aspectos metodológicos y las técnicas e instrumentos fundamentales para poder elaborar dichos trabajos, constituye la razón por la que hemos desarrollado el presente artículo.

Todo trabajo de investigación, especialmente uno histórico-demográfico donde se deben de conjugar aspectos diversos, requiere una meto-

\begin{abstract}
This paper intends to show the usefulness and importance of the parish records for works of historical and demographic character and provide an overview of the methodological approaches and tools to extract information from such records in order to develop a study on evolution of the population. To do this, the possible methods of exploitation of the sources, content analysis techniques and basic computer programs for their development are presented.
\end{abstract}

Keywords: Demography. Parish books. Analysis of records. Databases. Cáceres (Spain). Extremadura (Spain).

dología y técnicas que permitan la aprehensión de todos los elementos. Este tipo de estudios tiene como base principal una serie de fuentes sobre las que se fundamentan y que permiten su construcción. Así pues, en un estudio histórico-demográfico, las fuentes esenciales para su elaboración son aquellas que nos aportan información directa sobre la historia social de la población, por lo tanto, sobre la evolución de la mortalidad, natalidad y nupcialidad. De ahí que los registros parroquiales hayan sido el primordial pilar sobre el que se apoyan.

\subsection{Fuentes}

Los libros sacramentales constituyen un hito en la demografía, no solo por su condición de datos directos o por la abundancia, riqueza y rigurosa información que aporta sobre una población, sino también porque nos proporcionan una visión dinámica de las sociedades que permite seguir la evolución de las distintas variables, sus tendencias, ciclos, momentos de crisis y expansión.

Respecto a la información que aparece en las partidas podemos clasificarla en tres grupos distintos: 1) rasgos personalizadores -el nombre y los apellidos-; 2) rasgos primarios estado civil, padres, origen, vecindad, edad, fecha de ceremonia y grado de parentesco-; y 
3) rasgos complementarios -profesión, padrinos, causa de defunción, referencias al testamento, etc.-.

Desde un punto de vista de la Demografía Histórica, estos aspectos son útiles, pero no todos ellos nos sirven de manera directa para acercarnos al comportamiento de una sociedad. Así pues, los "rasgos principales" son los de mayor importancia porque nos aproximan a las distintas condiciones por las que pasan las sociedades a lo largo de los años, ya que nos ponen en relieve las crisis, los conflictos bélicos, así como los períodos de euforia o recuperación y los posibles cambios de mentalidad o mejoras socioeconómicas y sanitarias. Sin embargo, el primer y tercer tipo, aparte de aportarnos aspectos cualitativos, también nos ayudan a obtener datos significativos como el nivel económico de una persona, el número de hijos o la amplitud matrimonial.

\subsection{Metodología}

En demografía existen, principalmente, dos métodos para explotar la documentación parroquial: el análisis agregativo y el de reconstrucción de familias. Si bien es cierto que tanto uno como el otro tienen sus ventajas y desventajas, el más adecuado es el segundo ya que permite una explotación exhaustiva de las fuentes. Así pues, el primer método se caracteriza porque la recolección de los datos es rápida pero anónima lo que imposibilita acceder a datos esenciales, mientras que el método de reconstrucción consiste en recoger todos los datos de los tres libros sacramentales para reconstruir con ellos las familias de una población, lo que permite un conocimiento global de ella no solo desde un punto de vista demográfico sino también social.
En cualquier caso, antes de comenzar el vaciado sistemático de los distintos registros parroquiales, es necesario contar con fichas (Figura 1) que nos faciliten tanto la recogida como la lectura de los datos para poder analizarlos. El formato de la ficha dependerá, principalmente, del tipo del trabajo que queramos realizar. En todo caso, la labor de recogida de la información siempre hay que orientarla en función del objetivo a alcanzar. Por eso, las fichas de bautismos, matrimonios y defunciones se han de configurar para obtener datos precisos de cada ciclo vital y evitar una acumulación de información innecesaria.

\subsection{Instrumentos}

El empleo de programas informáticos constituye, sin duda, uno de los pilares de los estudios históricos-demográficos, ya que no solo permite agilizar el trabajo sino también plasmar los resultados de una manera excepcional.

En nuestro caso, solemos emplear tres programas informáticos para el tratamiento de los datos. Para el vaciado de los registros se utiliza la base de datos Filemaker Pro, un programa que aligera notablemente la recogida de información de las partidas sacramentales al concentrar los datos requeridos en un espacio visible. En segundo lugar, utilizamos, para la reconstrucción de las familias, el Excel, una hoja de cálculo que permite ordenar y filtrar la información. Además nos facilita el cálculo de medidas, tales como, la edad de acceso al matrimonio, concepción, defunción, intervalos genésicos, tasas, etc; así como, la elaboración de los gráficos primordiales para el análisis de las variables demográficas.

\begin{tabular}{|c|c|c|c|c|c|}
\hline Parroquia $\vdash$ & Hoyos & Nombre del marido & Faustino & Nombre de la mujer & Juliana \\
\hline Tipo de acta & Matrimonial & Apellido del marido & Álvarez & Apellido de la mujer & Gómez \\
\hline Número de registro 2 & 2573 & Origen del marido & Cilleros & Origen de la mujer & Hoyos \\
\hline $\begin{array}{l}\text { Matrimonio o } \\
\text { velación }\end{array}$ & & Profesión del marido & Militar & Profesión de la mujer & \\
\hline Fecha 0 & 05/10/2012 & Estado civil previo & so & Estado civil previo de la mujer & SA \\
\hline Parroquia de & Hoyos & Edad al matrimonio & & Edad al matrimonio de la mujer & \\
\hline matrimonio & & Nombre de la primera mujer & & Nombre del primer marido & \\
\hline Fecha del matrimonio 2 & $26 / 06 / 1822$ & Apellido de la primera mujer & & Apellido del primer marido & \\
\hline Grado de 0 & 0 & & & Profesión del primer marido & \\
\hline consanguinidad & & Nombre del padre del marido & Miguel Jorge & Nombre del padre de la mujer & Ventura \\
\hline Grado de afinidad 0 & & Apellido del padre del marido & Álvarez & Apellido del padre de la mujer & Gómez \\
\hline Información adiciomal e & el marido es subteniente de la milicia & Origen del padre del marido & Cilleros & Origen del padre de la mujer & Hoyos \\
\hline & & Profesión del padre del marido & & Profesión del padre de la mujer & \\
\hline & & Nombre de la madre del marido & Inés & Nombre de la madre de la mujer & Vicenta \\
\hline & & Apellido de la madre del marido & Pérez & Apellido de la madre de la mujer & Luengo \\
\hline & & $\begin{array}{l}\text { Profesión de la madre del } \\
\text { marido }\end{array}$ & & $\begin{array}{l}\text { Profesión de la madre de la } \\
\text { mujer }\end{array}$ & \\
\hline & & Origen de la madre del marido & Cilleros & Origen de la madre de la mujer & Hoyos \\
\hline & & $\begin{array}{r}\text { Residencia de los padres del } \\
\text { marido }\end{array}$ & Cilleros & $\begin{array}{l}\text { Residencia de los padres de la } \\
\text { mujer }\end{array}$ & Hoyos \\
\hline
\end{tabular}

Figura 1. Modelo de ficha 
Finalmente, hemos usado MapViewer, un programa de diseño cartográfico que permite hacer mapas de diversos tipos. Este instrumento es clave puesto que gracias a él podemos reflejar los flujos migratorios y analizar el mercado matrimonial, las distancias y diversos factores de gran importancia.

\subsection{Ejemplo práctico}

Para ilustrar todo lo que hemos expuesto anteriormente, vamos a recrear un caso donde se indica las pautas a seguir para hacer un trabajo histórico-demográfico. En este supuesto vamos a mostrar cómo analizar la nupcialidad de una población rural. Cabe señalar que aunque el ejemplo se centra en el empleo de fuentes parroquiales del Archivo Diocesano de CoriaCáceres, las bases metodológicas y conceptuales que se aportan son globales, y de aplicación a otros archivos.

Los pasos son los siguientes: a) vaciado de los registros parroquiales mediante el empleo de una base de datos; b) colocación y filtrado de la información en Excel; c) cálculo de las diferentes tasas a partir de las referencias que nos ofrecen las fuentes, y representación de los datos en gráficos o tablas; y d) Análisis de gráficos, tablas y datos.

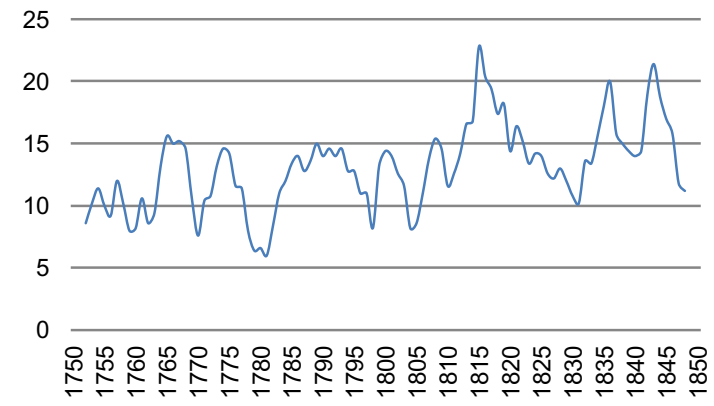

Figura 2. Evolución de la nupcialidad de Eljas, 1750-1850

Así pues, la indicación en las partidas matrimoniales de la fecha en la que se celebran los encales, nos sirve para trazar la línea evolutiva de la nupcialidad (Figura 2), la que nos mostrará los diferentes altibajos que se suceden a lo largo del tiempo en el seno de una población.

En esta misma línea argumental, si nos atenemos a una distribución mensual (Figura 3) verificamos como la elección de la fecha de matrimonio está determinada por factores económicos y religiosos (2). Por consiguiente, el mejor momento para casarse es en aquellos momentos cuando existe una menor actividad laboral, es decir, en noviembre, enero y febrero.

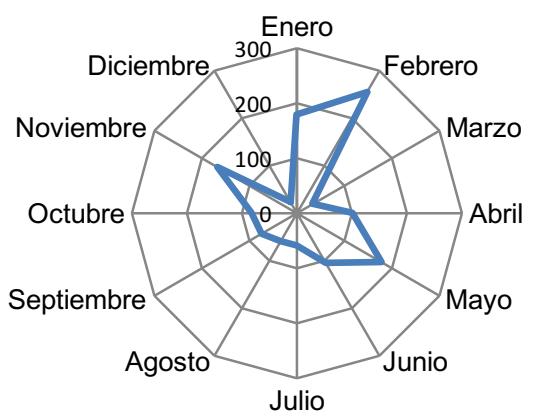

Figura 3. Estacionalidad de los matrimonios, 1750-1850

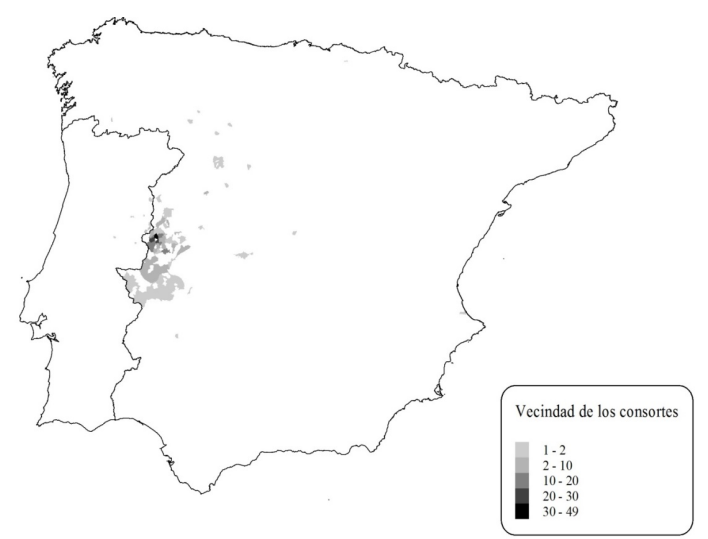

Figura 4. Procedencia geográfica de los consortes

Por otro lado, si analizamos la información referente al origen y vecindad de los consortes podemos conocer los movimientos migratorios así como las personas que conforman el mercado matrimonial y si éste es principalmente local, regional, nacional o internacional. Si observamos la Figura 4, se puede verificar como la mayoría de los cónyuges proceden de poblaciones cercanas, es decir, con aquellas con las que tienen una mayor relación motivada por intercambios comerciales. Por lo tanto, podemos comprobar que en función del parámetro que tomemos de los registros matrimoniales podemos analizar una dimensión de la nupcialidad, ya sea, su evolución, la edad nupcial, su estacionalidad, etc.

\section{Conclusión}

A lo largo de este trabajo hemos visto, por un lado, como a partir de las técnicas metodológicas expuestas es posible extraer los datos, tanto explícitos como implícitos, de los libros sacramentales. Por lo tanto, consideramos que tanto la metodología como los instrumentos son de gran utilidad para conseguir nuestro objetivo. 
Por otro lado, hemos verificado que los libros parroquiales constituyen la fuente por excelencia de los estudios históricos-demográficos, puesto que la información que contienen nos permiten analizar los diversos aspectos de una población, a la vez, que reflejan los problemas sociales y económicos por los que atraviesa a lo largo de los años.

\section{Notas}

(1) Un excelente estudio sobre la evolución de la Demografía Histórica es el trabajo de Saito, Osamu (1997).

(2) La Iglesia, tras el concilio de Trento, prohíbe la celebración de matrimonios como símbolo de penitencia y austeridad entre Cuaresma y el Domingo de Quasimodo y de Adviento a Epifanía.

\section{Referencias}

Amorim, María Noberta (1991). Uma metodología de reconstituição de paróquias. Universidade do Minho.

Bradbrook, W. (1910). The Parish Registers. London.

Burke, A.M. (1908). Key to the Ancient Parish Registers of England an Wales. London.

Cardono, Ciro; Pérez Brignoli, H. (1986), La explotación de los registros parroquiales, en Los métodos de la Historia. Barcelona: Editorial Crítica Grijalbo.

Cox, J. C. (1910), The Parish Registers of England. London.

Dupaquier, Jacques (1972). Methode d'interpretation des sources non nominatives par la construccion de modeles// Annales de Demographie Historique. 180-188.

Dupaquier, Jacques (1974). Introduction à la démographie historique. Paris-Tournia-Montreal.

Eversley, D.E.C. (1966). Exploitation of Anglican Parish Registers by Aggregative Analysis. // E. A. Wrigley (ed.), An Introduction to English Historical Demography, London, 61-65.

Fernández Catón, José María (dir.) (1978). Los archivos de la Iglesia de España. León.

Fleury, Michel; Henry, Louis (1985). Noveau manuel de dépovillement et d'explotation de L'etat civil ancien. París: Institut national d'études démographiques.

Goubert, Pierre (1954), Une richesse historique en cours d'exploitation: les registres paroissiaux. I/ Annales E.S.C., 9:1, 83-93.

Henry, Louis; Fleury, Michel (1956). Des registres paroissiaux à l'histoire de la population. Manuel de dépouillement et d'exploitation de l'état civil ancien. París: Institut d'Etudes Démographiques.

Jedin, Hubert (1943). Le origini dei registri parrochiali e il Concilio de Trento. // II Concilio de Trento, T.2. RomaMilano, 323-336.

Le Mée, René (1995). De la naissance de la démographie historique à l'enquête Henry // Population. 6, 1475:1488.

Levron, J (1959), Les registres paroissiaux et d'etat civil en France. // Archivum. 55-100.

Livi Bacci, Massimo (1993). Introducción a la demografía. Barcelona: Arial.
Martín Galán, Manuel Mariano (1981). Fuentes y métodos para el estudio de la Demografía Histórica castellana durante la Edad Moderna. // Hispania. 41:148, 231-326.

Mols, Roger (1954-56). Introduction à la démographie historique del villes d'Europe du XVle au XVIIle siècle. Louvain. 3 vols.

Nadal, Jordi (1992). Bautismos, desposorios y entierros. Estudios de Demografía Histórica. Barcelona: Ariel.

Pérez Puchal, Pedro (1973). Fuentes y métodos la Demografía Histórica. // Estudios Geográficos. 34:130, 5-32.

Pérez Moreda, Vicente; Reher, David- Sven, (eds.) (1988). Demografía Histórica en España. Madrid: Arquero.

Pursen, J.; Valdearcos, E.; et al. (1986), La población de Zaragoza en el siglo XVIII, un ejemplo de mecanización de registros parroquiales. // Ubieto Arteta, Agustín (coord.). Metodología de la investigación científica sobre fuente aragonesas: actas de las I Jornadas celebradas en Monzón, del 8 al 20 de diciembre de 1985, 83-92.

Rasi, .P (1939). Note per la storia dei registri di stato civile. // Studi di Sotria e Diritto inore di Enrico Besta, T. III, Milano, 465-491.

Reher, David-Sven (2000). La investigación en demografía histórica: pasado, presente y futuro. // Boletín de la Asociación Demográfica Histórica. 18:2, 15-78.

Rodríguez Calleja, Jesús Emiliano (s. d.). Metodología para la explotación de los registros parroquiales. // Revista digital cuarto de apero. http://cuartodeapero.com/re sources/_Metodolog\$C3\$ADa.doc_.pdf (2016-03-19).

Rosental, Paul-André (1996). Treize ans de reflexion: de l'histoire des populations à la démographie historique (France, 1945-1985). // Population. 6, 1211:1238.

Rueda Fernández, José Carlos (1990). Los Registros Parroquiales en Castilla: notas sobre su implantación, desarrollo y reglamentación en la ciudad y diócesis de Zamora (siglos XVI-XVII). // Studia historica. Historia Moderna. 8, 7-37.

Saito, Osamu (1997). Demografía histórica: Realizaciones y expectativas. // Revista de Demografía Histórica. 15:1,169-204.

Salas, José Antonio (1986), Los "registros parroquiales" y sus posibilidades como fuentes históricas. // Ubieto Arteta, Agustín (coord.). Metodología de la investigación científica sobre fuentes aragonesas: acta de las I Jornadas celebradas en monzón, del 8 al 20 de diciembre de 1985, 21-72.

Salas Auséns, José Antonio (1975-1977). Los registros parroquiales como fuente para el estudio de la inmigración. Franceses en Barbastro entre dos centurias (siglos XVI-XVII). // Argensola: Revista de Ciencias Sociales del Instituto de Estudios Altoaragoneses, 79-84, 67-84.

Shryock, Henry S.; Siegel, Jacob S.; et al (1976). The methods and materials of demography. Washington: Elizabeth $A$. Larmon.

Willigan, J. D. y Lynch, K.A. (1982), Sources and Methods of Historical Demography, New York.

Wrigley, Edward Anthony (1985). Historia y población. Introducción a la demografía histórica. Barcelona: Crítica.

Enviado: 2016-03-27. Segunda versión: 2017-05-09. Aceptado: 2017-05-09. 